\title{
Synovial Sarcoma of Hand Presenting as a Cystic Mass
}

\author{
Chbani Laila ${ }^{1}$, Badioui Ikram', Shimi Mohamed², Daoudi Abdelkrim², Tizniti Siham³, \\ Mrini Abdelmajid ${ }^{2}$, Amarti Afaf ${ }^{1}$ \\ ${ }^{1}$ Department of Pathology, Hassan II Teaching Hospital, Fez, Morocco; ${ }^{2}$ Department of Orthopedic Surgery, Hassan II Teaching \\ Hospital, Fez, Morocco; ${ }^{3}$ Department of Radiology, Hassan II Teaching Hospital, Fez, Morocco. \\ Email: chbanil@yahoo.fr
}

Received March $8^{\text {th }}$, 2012; revised April 5 ${ }^{\text {th }}, 2012$; accepted May $17^{\text {th }}, 2012$

\begin{abstract}
Synovial sarcoma is a rare mesenchymal tumor. It occurs predominantly in the extremities especially in the region of the knee. The localization in palm hand is rare. We report a case of a 23 year-old man who present a monophasic synovial sarcoma of the left hand which presented as a painless cystic nodule. A surgical excision was performed. The result was good without recurrence. The synovial sarcoma is a rare malignant neoplasm that should be considered in the differential diagnosis of spindle neoplasms of hand.
\end{abstract}

Keywords: Synovial Sarcoma; Hand; Differential Diagnosis

\section{Introduction}

Synovial sarcoma is a rare malignant neoplasm [1]. The palm hand is a very rare location. Because of the similar radiological and histological features, these tumors can be confused with benign lesions such as giant cell tumors. We present a case of monophasic synovial sarcoma arising in the palm hand, which presented as a subcutaneous nodule.

\section{Clinical Case}

A 23 year-old right-handed men presented with painful mass of his left palm hand. He had no history of trauma or constitutional symptoms such as fever. Physical examination revealed a $5 \mathrm{~cm}$ mass on the palmar region of the left hand. There was no dermal involvement or ulceration. The mass was not tender or pulsatile. The MRI (magnetic resonance imagery) (Figure 1) examination revealed a well-demarcated subcutaneous cystic mass appeared heterogeneous on the T1-weighted image. The diagnosis of giant cell tumour of tendon sheath was proposed.

Tumor excision with large margin was performed under brachial-plexus block anesthesia. Gross examination showed a cystic mass, measuring $5 \times 5 \times 3 \mathrm{~cm}$, composed of pale, yellow-white tissue as observed at the cut surface. Microscopic sections revealed monotonous spindle cells with elongated nuclei and little cytoplasm (Figure 2). There was not much stroma between the cells, but focally there were areas of thick bundles of collagen. There were 11 mitoses per high-power fields. The he- mangiopericytic vascularization was also noted (Figure 3).

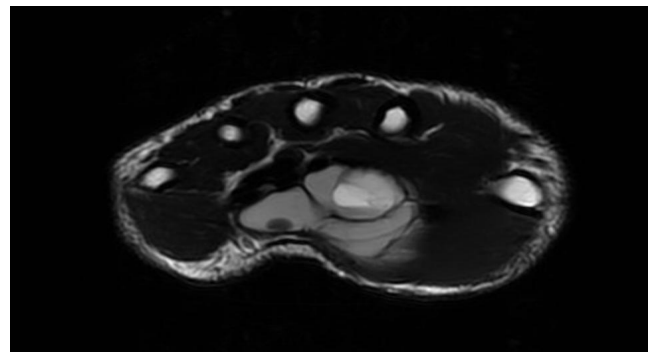

Figure 1. Magnetic resonance images showing the palmar cystic mass.

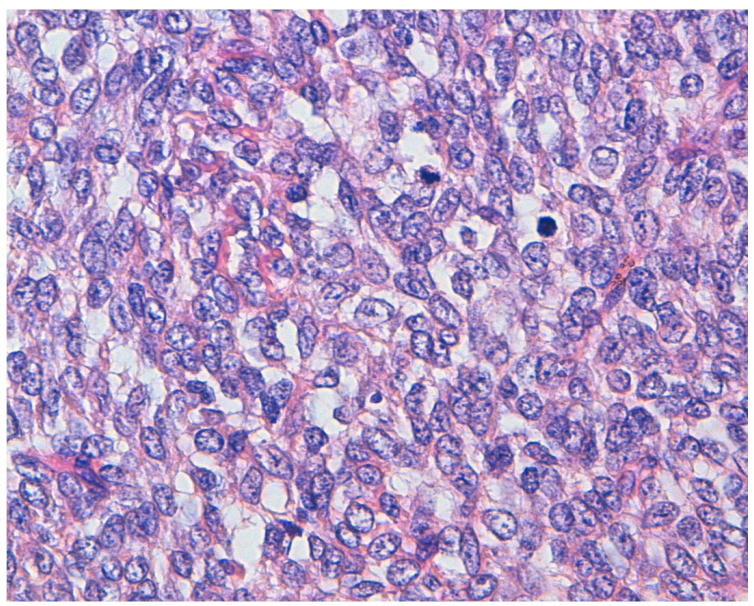

Figure 2. Dense proliferation of spindle cells with mitoses (hematoxylin and eosin $\times 400$ ). 


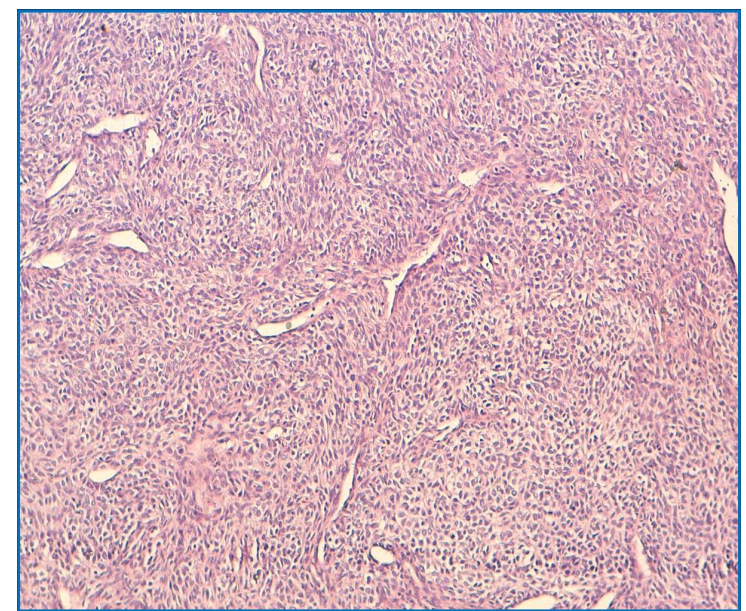

Figure 3. Hemangiopericytomatous vascular pattern (hematoxylin and eosin $\times 250$ ).

The lesion contained no necrosis areas or giant cells. Immunohistochemistry showed that the neoplastic cells were negative for cytokeratin, epithelial membrane antigen, smooth muscle actin and S-100 protein. The diagnosis of synovial sarcoma was proposed.

Molecular study using reverse transcription-PCR was performed. The SYT-SSX2 fusion transcript associated with the $t(\mathrm{X}, 18)$ translocation typical of synovial sarcoma was found.

Approximately 12 months after the operation, there was no clinical or radiographic evidence of recurrence or metastasis.

\section{Discussion}

Synovial sarcoma is a rare and aggressive sarcoma accounting for approximately $5 \%$ of malignant soft tissue tumors [1] usually occurring in young adults between the ages of 15 and 40 years and rarely may occur in children [2]. The most common sites include lower extremities $(60 \%)$, with predilection for the knee area; upper extremities (23\%); the head and neck region (9\%). It is rarely seen in the hand [3]. Lotz et al. reported 107 cases of synovial sarcoma of the hand located less frequently in the digits than in the carpus.

In this location, these tumors seem to follow a clinically favorable course [4].

The origin of this is unclear. As joint involvement is very uncommomn and as it may arise in synovium lacking organs, it is thought that synovial sarcoma does not arise from synovial cells but from an undefined mesenchymal cell, able to have a double epithelial and spindle cell differentiation $[1,2]$.

Two major histologic subtypes exist: monophasic and biphasic synovial sarcomas. These subtypes are determined by the presence or absence of epithelial components without difference in prognosis between these his- tologic subtypes.

The monophasic synovial sarcoma; like in our case; is characterized by the presence of spindle cells with collagenous stroma and hemangiopericytomatous vascular pattern.

Positive immunohistochemical staining for cytokeratine or epithelial membrane antigen helps to confirm the diagnosis of monophasic synovial sarcoma. Other markers, including Bcl2 and CD99 may display positivity.

This immunohistochemical analysis can help to distinguish synovial sarcoma to other mesenchymal neoplasms such as palmar fibromatosis, fibroblastic sarcoma and giant cell tumors in fusocellular variant.

Presenting as a cystic mass, the synovial sarcoma can be confused with other causes of cystic mass of hand like synovial cyst or mucoid cyst who contain mucin. A fibrous capsule and myxomatous stroma interspersed with fibroblasts surround the cyst without mitose.

Genetically, synovial sarcomas are characterized by the recurrent presence of a specific chromosomal translocation, $t(\mathrm{X}, 18)$ (p11; q11), wich usually results in 1 of 2 fusion genes: SYT-SSX1 or SYT-SSX2. The identification of these chimeric gene seems to be useful tool for the differential diagnosis and for the prognosis since SYT-SSX2 [5] associated mostly with monophasic types and a significantly better prognosis like in our case.

The treatment of synovial sarcoma of hand is surgical consisting of excision with large margin.

Synovial sarcomas have been reported to recur and metastasize. Metastasis has also been reported to occur frequently with lung being a common site. In our case, the patient had no evidence of local recurrence or metastasis after 12 months.

\section{Conclusion}

Synovial sarcoma should be considered in the differential diagnosis of cystic lesions involving the hand.

\section{REFERENCES}

[1] C. Fisher, "Synovial Sarcoma," Annals of Diagnostic Pathology, Vol. 2, No. 6, 1998, pp. 401-21. doi:10.1016/S1092-9134(98)80042-7

[2] C. Fisher, D. R. H. de Bruijn and A. Geurts van Kessel, "Synovial Sarcoma," In: C. Fletcher, K. K. Unni and F. Mertens, Eds., World Health Organization Classification of Tumours, Pathology and Genetics of Soft Tissue and Bone, International Agency for Research on Cancer Press, Lyon, 2002, pp. 200-204.

[3] S. Lotz, J. Caselitz, J. Bullerdiek and K.-U. Rieckhoff, "Monophasisch Fibroses Synovialsarkom der Hand Mit Biphasisch Differenzierten Lungenmetastasen,” Pathologe, Vol. 14, No. 1, 1993, pp. 54-57.

[4] M. Michal, J. C. Fanburg-Smith, J. Lasota et al., "Minute Synovial Sarcomas of the Hands and Feet: A Clinicopa- 
thologic Study of 21 Tumors Less than $1 \mathrm{~cm}$,” American Journal of Surgical Pathology, Vol. 30, No. 6, 2006, pp. 721-726. doi:10.1097/00000478-200606000-00007

[5] A. Kawai, J. Woodruff, J. H. Healey, M. F. Brennan, C. R. Antonescu and M. Ladanyi, "SYT-SSX Gene Fusion as a
Determinant of Morphology and Prognosis in Synovial Sarcoma," The New England Journal of Medicine, Vol. 338, No. 3, 1998, pp. 153-160. doi:10.1056/NEJM199801153380303 